\title{
Physical Properties of Fullerene-containing Galactic Planetary Nebulae
}

\author{
M. Otsuka*1, F. Kemper ${ }^{1}$, J. Cami ${ }^{2,3}$, E. Peeters ${ }^{2,3}$, J. Bernard-Salas ${ }^{4}$ \\ ${ }^{1}$ Institute of Astronomy and Astrophysics, Academia Sinica, $11 \mathrm{~F}$ of Astronomy-Mathematics \\ Building, AS/NTU. No.1, Sec. 4, Roosevelt Rd, Taipei 10617, Taiwan, R.O.C. \\ ${ }^{2}$ Department of Physics and Astronomy, The University of Western Ontario, London, ON N6A \\ 3K7, Canada \\ ${ }^{3}$ SETI Institute, 189 Bernardo Ave, Suite 100, Mountain View, CA 94043, USA \\ ${ }^{4}$ Department of Physical Sciences, The Open University, Milton Keynes, MK7 6AA, UK \\ E-mail: btsukadasiaa.sinica.edu.tw
}

\begin{abstract}
We searched the Spitzer Space Telescope data archive for Galactic planetary nebulae (PNe), that show the characteristic 17.4 and $18.9 \mu \mathrm{m}$ features due to $\mathrm{C}_{60}$, also known as buckminsterfullerene. Out of 338 objects with Spitzer/IRS data, we found eleven $\mathrm{C}_{60}$-containing $\mathrm{PNe}$, six of which (Hen2-68, IC2501, K3-62, M1-6, M1-9, and SaSt2-3) are new detections. The strongest 17.4 and $18.9 \mu \mathrm{m} \mathrm{C}_{60}$ features are seen in Tc 1 and SaSt 2-3, and these two sources also prominently show the $\mathrm{C}_{60}$ resonances at 7.0 and $8.5 \mu \mathrm{m}$. In the other nine sources, the 7.0 and $8.5 \mu \mathrm{m}$ features due to $\mathrm{C}_{60}$ are much weaker. The flux ratio between the 17.4 and $18.9 \mu \mathrm{m} \mathrm{C}_{60}$ feature is rather constant amongst the sample, with an average value of 0.49 . We find that the Polycyclic Aromatic Hydrocarbon (PAH) profile over 6-9 $\mu \mathrm{m}$ in these $\mathrm{C}_{60}$-bearing carbon-rich $\mathrm{PNe}$ is of the more chemically-processed class A. The Spitzer spectra also show broad dust features around 11 and $30 \mu \mathrm{m}$. The strength of the $11-\mu \mathrm{m}$ feature is correlated to the temperature of the dust, suggesting that it is at least partially due to a solid-state carrier.
\end{abstract}

The Life Cycle of Dust in the Universe: Observations, Theory, and Laboratory Experiments 18-22 November, 2013

Taipei, Taiwan

\footnotetext{
${ }^{*}$ Speaker.
} 

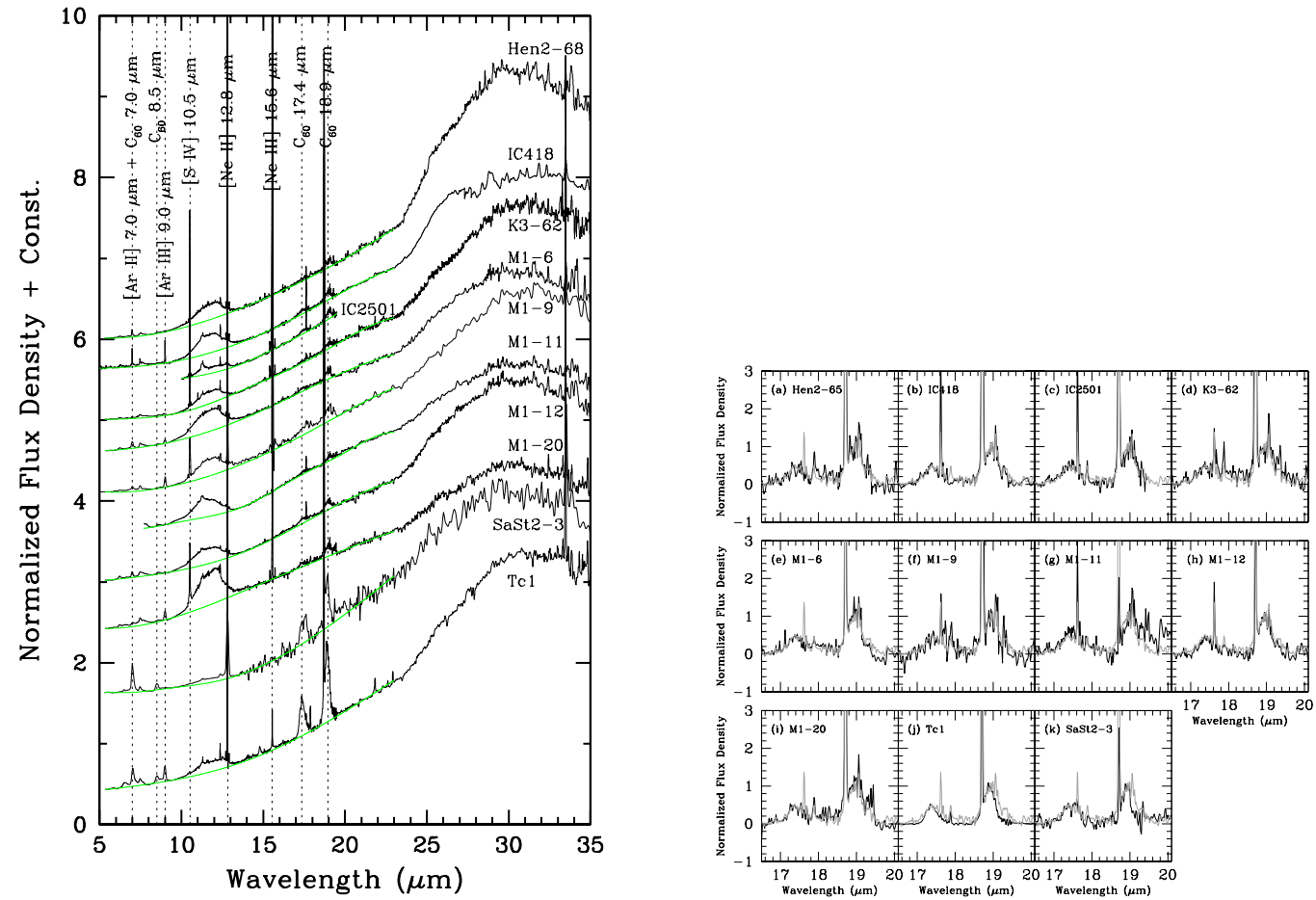

Figure 1: (left) The 5.3-35 $\mu \mathrm{m}$ Spitzer/IRS and ISO spectra normalized to the flux density at $20 \mu \mathrm{m}$. The green lines are base lines determined by fitting the underlying feature-less continuum with third to fifth order spline in the range from 5.3 to $23 \mu \mathrm{m}$, except for IC2501 and M1-11. For IC2501 we determined the continuum over 2.5-5.0 $\mu \mathrm{m}$ and 10-19.6 $\mu \mathrm{m}$, while for M1-11 we used 2.5-5.0 and 8-23 $\mu \mathrm{m}$. The flux density normalization for IC2501 was using the extrapolated continuum from 10-19.6 $\mu \mathrm{m}$ continuum (the green line). (right) The local continuum subtracted spectra over 16.5-20.1 $\mu \mathrm{m}$, normalized to the peak flux density in the $18.9 \mu \mathrm{m} \mathrm{C}_{60}$ band. The black lines represent the observed spectra of each object and the grey lines show the average spectrum among all objects.

\section{Results}

We summarize our analyses of the Spitzer/IRS and ISO/SWS mid-infrared spectra of $\mathrm{C}_{60} \mathrm{PNe}$ as follows. The detailed discussion is found in Otsuka et al. (2014) [四]. The broad $30 \mu \mathrm{m}$ feature in our sample is discussed by Kemper et al. [[] in these proceedings.

Spectral Features and Variations in 5-36 $\mu \mathrm{m}$ The 5.3-35 $\mu \mathrm{m}$ spectra are presented in Figure 1 left. There are strong variations in the intensity of the $8.5,17.4$, and $18.9 \mu \mathrm{m} \mathrm{C}_{60}$ bands relative to the local dust continuum indicated by the green lines, with SaSt2-3 and Tc1 showing especially strong 8.5, 17.4, and $18.9 \mu \mathrm{m} \mathrm{C}_{60}$ bands. The 6-9 $\mu \mathrm{m} \mathrm{PAH}$ bands are seen in all PNe, although the band-profiles are different from ones frequently seen in non- $\mathrm{C}_{60} \mathrm{PNe}$. The broad 11 and 30 $\mu \mathrm{m}$ features are also seen in all PNe. All the spectra show a thermal dust continuum. Otsuka et al. (2014) [四] show that the dust continuum slope is different for the different objects, pointing to different dust temperatures, caused by the difference in irradiation (see their Figure 3). They found that the strengths of the $17.4 \mu \mathrm{m}$ and $18.9 \mu \mathrm{m} \mathrm{C}_{60}$ bands are not changing with the local dust continuum. 


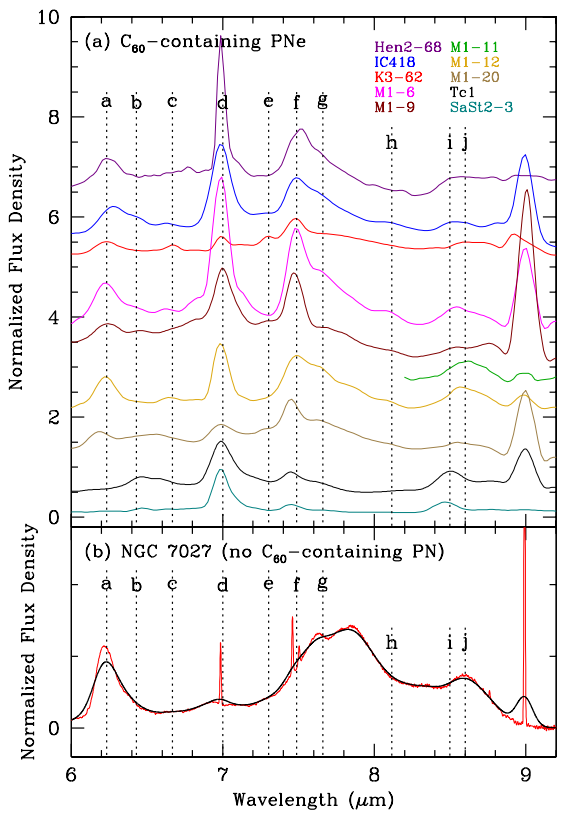

Figure 2: (upper panel) The continuum subtracted over 6.0-9.2 $\mu \mathrm{m}$ PAH line-profiles of $\mathrm{C}_{60} \mathrm{PNe}$. (lower panel) The ISO/SWS spectrum of the C-rich PN NGC7027. The high-excitation gas lines are subtracted out. The resolution of this spectrum (red line) was reduced to that of Spitzer/IRS (black line). The emission features seen in these spectra are indicated by dotted lines and lower-case letters. a: $\mathrm{PAH} \mathrm{C}=\mathrm{C}$ stretch 6.23 $\mu \mathrm{m}, \mathrm{b}: \mathrm{C}_{60}^{+} 6.4 \mu \mathrm{m}$ (possible), c: $\mathrm{C}_{24}$ planar (possible) or PAH C=C stretch $6.67 \mu \mathrm{m}, \mathrm{d}: \mathrm{C}_{60} 7.0 \mu \mathrm{m}+$ [Ar II] $6.99 \mu \mathrm{m}$, e: an emission feature at $7.30 \mu \mathrm{m}$ seen by Scott \& Duley (1996) [3] and Scott et al. (1997) [四], f: $\mathrm{C}_{60}^{+}+\mathrm{H}$ I complex, g: PAH C-C stretch $7.7 \mu \mathrm{m}, \mathrm{h}: \mathrm{C}_{60}^{+} 8.1 \mu \mathrm{m}$ (possible), i: $\mathrm{C}_{60} 8.5 \mu \mathrm{m}$, j: PAH C-H in-plane bend $8.6 \mu \mathrm{m}$.

The 17.4 and 18.9 $\mu \mathbf{m ~ C}_{60}$ line profiles Figure 1 right displays the continuum subtracted spectra (black lines), showing the 17.4 and $18.9 \mu \mathrm{m} \mathrm{C}_{60}$ bands. The normalized line profiles of the 17.4 and $18.9 \mu \mathrm{m} \mathrm{C}_{60}$ bands are similar to each other, as can be seen from the average spectrum (grey line). The flux ratio between the 17.4 and $18.9 \mu \mathrm{m} \mathrm{C}_{60}$ feature is rather constant amongst the sample, with an average value of 0.49 .

The 7.0 and $8.5 \mu \mathbf{m ~ C}_{60}$ and the broad 6-9 $\mu \mathrm{m}$ complex The line-profiles are similar to each other, except for the 6.2 and $8.5 \mu \mathrm{m}$ bands (Figure 2). There is the possibility that the 6.99 and $7.07 \mu \mathrm{m} \mathrm{C}_{70}$ resonances might contribute to the $7.0 \mu \mathrm{m}$ band. However, we could not detect any $\mathrm{C}_{70}$ bands in our sample, except for the already reported features in Tc1 (Cami et al. 2010 []]). Therefore, in the PNe in our sample except for Tc1, the $7.0 \mu \mathrm{m}$ line is concluded to be due to a complex of the $6.92 \mu \mathrm{m}$ aliphatic vibration band, the [Ar II] $6.99 \mu \mathrm{m}$, and the $7.0 \mu \mathrm{m} \mathrm{C}_{60}$ band. Tc1 and SaSt2-3 clearly show the strong $8.5 \mu \mathrm{m} \mathrm{C}_{60}$ band, while the $6.2 \mu \mathrm{m}$ PAH band is very weak. In other sources these bands are heavily contaminated by PAHs at 8.6 and $6.2 \mu \mathrm{m}$, respectively. The 7.2-8.1 $\mu \mathrm{m}$ band profiles are quite different from that in a Class B PAH C-rich PN NGC7027. Class B PAH objects exhibit the emission peak at $\sim 7.8-8 \mu \mathrm{m}$ (Peeters et al. 2002 [四]), while $\mathrm{C}_{60}$ PNe emit the bulk of their emission around $\sim 7.7 \mu \mathrm{m}$. Hence, according to the classification of the 6-9 $\mu \mathrm{m}$ PAH profiles, all $\mathrm{C}_{60}$ PNe exhibit Class A PAH profiles. The carrier of 

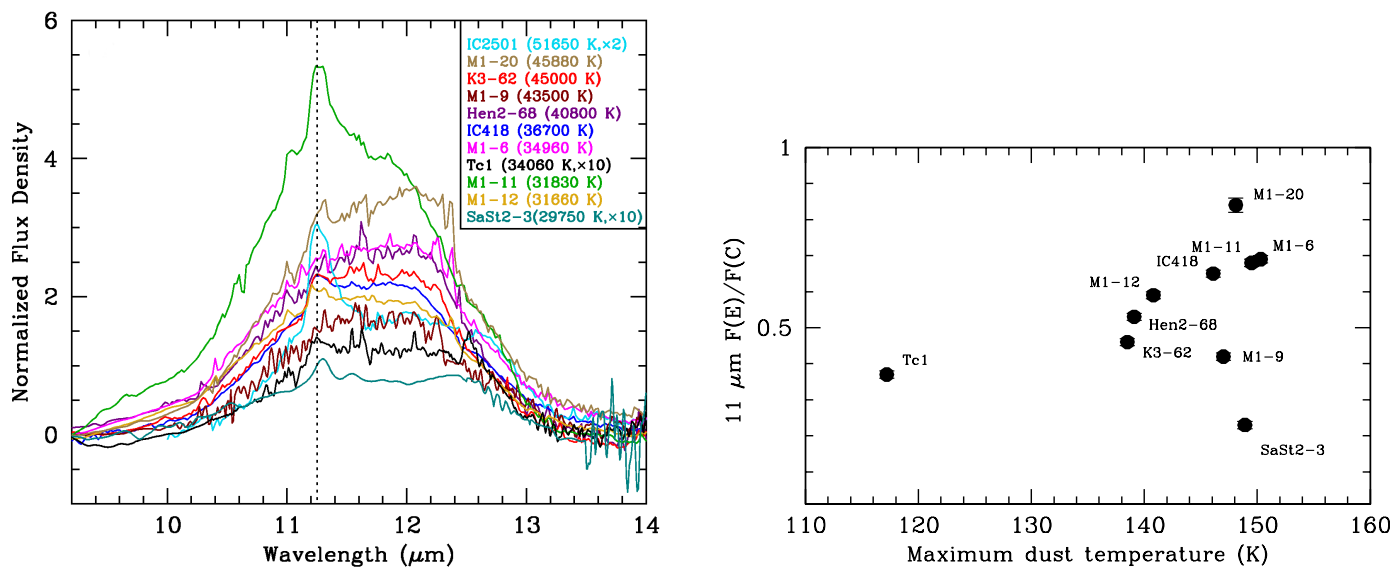

Figure 3: (left) The continuum subtracted spectra over 9.1-14 $\mu \mathrm{m}$, normalized to the peak flux density of $\mathrm{C}_{60} 18.9 \mu \mathrm{m}$, except for IC2501, SaSt2-3, and Tc1. The scaling factor for these PNe are 2, 10, and 10 after the normalization to $\mathrm{C}_{60} 18.9 \mu \mathrm{m}$. The effective temperatures of the PN central stars are listed in the upperright corner. The position of the $11.3 \mu \mathrm{m}$ PAH is indicated by the dotted line. (right) The plot between the band strength of the broad $11 \mu \mathrm{m}$ band and the maximum dust temperature.

the Class A is believed to be more chemically processed than that of the Class B profiles.

The broad $11 \mu \mathrm{m}$ feature The emission profiles show a nearly flat portion between 11.2 and 12.4 $\mu \mathrm{m}$, except for M1-11 (Figure 3 left). The peak intensity of the $11 \mu \mathrm{m}$ feature in SaSt2-3 and Tc1, which show the prominent $\mathrm{C}_{60}$ bands, is much weaker than in the other PNe. A correlation between the $11 \mu \mathrm{m}$ band strength and the maximum dust temperature, suggests that the carrier of this feature is a thermally heated dust component (Figure 3 right). On the other hand, the substructure in the $11 \mu \mathrm{m}$ feature points to a carrier related to PAHs. Kwok et al. (2001) [四] argue that the $11 \mu \mathrm{m}$ feature is a collection of out-of-plane bending modes of aliphatic side groups attached to aromatic rings. Further discussion about the carrier is necessary.

\section{References}

[1] M. Otsuka et al. 2014 Physical properties of fullerene-containing Galactic planetary nebulae, MNRAS, 437, 2577

[2] F. Kemper et al, On the nature of the 30 micron feature in carbon-rich planetary nebula, in proceedings of The Life Cycle of Dust in the Universe: Observations, Theory, and Laboratory Experiments, POS (LCDU2013) 122 .

[3] A. Scott and W. W. Duley 1996 The Decomposition of Hydrogenated Amorphous Carbon: A Connection with Polycyclic Aromatic Hydrocarbon Molecules, ApJL, 472, L123

[4] A. D. Scott et al. 1997 Infrared Emission Spectra from Hydrogenated Amorphous Carbon, ApJL, 490, L175

[5] J. Cami et al. 2010 Detection of $C_{60}$ and $C_{70}$ in a Young Planetary Nebula, Science, 329, 1180

[6] E. Peeters et al. 2002 The rich 6 to $9 \mu \mathrm{m}$ spectrum of interstellar PAHs, A\&A, 390, 1089

[7] S. Kwok et al. On the Origin of Infrared Plateau Features in Proto-Planetary Nebulae, ApJL, 554, L87 